\title{
Preliminary experience on subcutaneous venous access device (chemoport) in a teaching hospital in Kathmandu
}

\section{Joshi R}

Robin Joshi, Assistant Professor, Kathmandu Medical College Teaching Hospital, Kathmandu, Nepal

\begin{abstract}
Background: Modern subcutaneous venous access device or chemoport nowadays is a vital device used in case of chronic diseases. It is now an established device for administration of medication and blood withdrawal without difficulty and negating repeated skin punctures for those patients who need repeated and long term intravenous medication. But, these devices are not without complications.

Objective: To assess preliminary technical success and complication rates of the ultrasound and fluoroscopy guided placement of subcutaneous venous access device.

Methods: Between November 2012 to May 2015, 10 port catheter components were implanted. All components were inserted under image guidance. Ultrasound guided puncture of right internal jugular vein was preferred and position of tip of catheter was confirmed by fluoroscopy. Early and late complications were evaluated. The overall cost of the chemoport was also taken into consideration

Results: The implantation was inserted in ten cases. Four patients had ovarian carcinoma, three patients metastatic colonic carcinoma, one had testicular cancer with mediastinal mass, one patient had metastatic invasive urinary bladder carcinoma and another one had porphyria whose peripheral venous access were all thrombosed due to repeated puncture. Peri-procedural early complications like blockage, thrombosis, leak were not observed, however one patient had catheter related fever. Late complications like blockage, port, fractures, dislodgement, venous thrombosis were also not found but One patient had port pocket infection. The cost of the port device in all cases was about 50,000 Nepalese rupees.

Conclusion: Larger number of case are required for better statistical evaluation.The main reason for the refusal of subcutaneous venous access device insertion by the patients was the high cost of the device. Cather-related morbidity was in acceptable range so chemoport insertion is feasible in Nepal. Major life threatening complication was not observed.
\end{abstract}

Key words: Chemoport, Subcutaneous venous access device

\section{INTRODUCTION}

hemoport or Portocath is an implantable Subcutaneous Venous Access Device (SVAD). Chemoport is inserted in patients requiring long term venous access. It can be kept for two years. It is now safe, easy and cosmetically acceptable procedure ${ }^{1}$. Chemoport can be inserted to right internal jugular vein (IJV) or right subclavian vein. Many centers prefer right IJV as the first choice ${ }^{1,2}$ as complication like pneumothorax can be avoided.

Address for correspondence

\section{Dr. Robin Joshi}

Assistant Professor, Department of Surgery

Kathmandu Medical College Teaching Hospital

Sinamangal, Kathmandu, Nepal

Email: robinjoshi@hotmail.com
Intervention radiologist and surgeons perform chemoport insertion. Use of ultrasound and fluoroscopy during the insertion has yielded good success rate and low peri-procedural complication ${ }^{3}$. Internal jugular vein is preferred as an access site using ultrasound guidance. Use of fluoroscopy helps to locate the site, correct placement of tip of catheter at cavoatrial junction ${ }^{4}$. As the IJV is located away from lung and it nerve plexus the stent related complications during insertion is less as compared to subclavian approach ${ }^{2,5,6}$ and complication like pneumothorax is avoided. We report our preliminary ten cases in Nepal from a teaching hospital. The objective was to assess preliminary technical success and complication rates of the ultrasound and fluoroscopy guided placement of subcutaneous venous access device via the internal jugular vein. 


\section{METHODS}

This is a study of ten chemoport inserted from November 2012 to May 2015. Verbal and written consents were obtained from all the patients. The chemoport insertions were done in operation theatre under aseptic condition. Catheter related immediate and early complications were recorded including mortality within 30 days of insertion. With no such catheter-related complications the procedure can be regarded as successful. Many patient receive disease related chemotherapy and mortality related due to disease process after the insertion of chemo-port is not regarded as catheterrelated mortality. Total cost including cost of device, operation charges were evaluated in this study. All insertions were performed under the aid of ultrasound and fluoroscopy.

The implantable port system was mainly of 6.58.5 Fr in size. We used the Bard access port system (Figure 1) in all patients. The system has a siliconebased catheter with non-valve titanium port. For preoperative antibiotic prophylaxis, one gram Ceftriaxone was given intravenously half an hour prior to the procedure. Abnormal coagulation profile with abnormal prothrombin time and activated partial thromboplastin time, international normalized ratio (INR) of more than 1.5 and platelet count of less than 50,000 were the relative contraindication and therefore patients with such abnormality were not included. Bacteraemia and sepsis are absolute contraindications for such procedure thus patients with such conditions were also excluded. Nine of the patients were oncology patients requiring chemoport for chemotherapy administration. One patient was diagnosed to have porphyria and needed repeated vein puncture for medication due to which all his peripheral venous access sites were thrombosed. He also needed repeated change of central venous access for medication.

\section{SURGICAL TECHNIQUE:}

The technique of insertion is like a central vein catheter insertion. Continuous blood pressure and pulse oximeter monitoring of the patients was made mandatory. No intravenous sedation was used in our cases. Skin preparation was done with povidone-iodine solution and sterile draping was used. An ultrasound guidance puncture of right internal jugular vein using a 19-G puncture needle (Figure 2,3) was done. After successful puncture, 0.018 -inch guidewire was inserted and position was confirmed by fluoroscopy. Then, an 8.5 or 10 French peel-away sheath was inserted through which catheter is inserted and position confirmed by fluoroscopy. The catheter was washed with dilute Heparin and clamped till the port reservoir was made. The port reservoir pocket was created about $2-3 \mathrm{~cm}$ below the ipsilateral clavicle. The port catheter was tunneled to the pocket and connected to the silicon reservoir. It was then fixed to the pocket by $2-0$ vicryl. The catheter tip was reconfirmed at the level of the cavoatrial junction or 2-3 cm below the carina correlating with second intercostal space using fluoroscopy. Aspiration of blood was done to check its function and flushed with heparinized saline. The port was secured to the pocket using absorbable suture then closed in two layers using absorbable polyglactin suture. Sterile dressings were placed. A post-procedural chest radiograph was taken (Figure 4). After the insertion, the patients were followed up on 7-10 days to check the wound site and functioning of the port. Periprocedural complications were divided into immediate, early and late. Immediate complications are during the procedure. Early complications are noted that arise within 24 hours to 30 days. Late complications are recorded after 30 days post-insertion.

\section{RESULTS}

The SVAD was inserted in 10 cases. There were six male and four female patients. The age of the patient ranged from 25-62 years and mean age was 50.5 years. Four female, three male, one male, and one male patients were diagnosed to have ovarian carcinoma, colonic carcinoma, invasive bladder carcinoma, testicular carcinoma respectively. All these patients were operated cases. The porphyric patient needed SVAD implantation for his regular intravenous medication and the peripheral veins were all thrombosed due to repeated punctures. This patient succumbed to his medical cause (Porphyria) on $17^{\text {th }}$ day of implantation.

Per-operative complication (Table 2) was not observed. Early complication like catheter-related blockage, thrombosis, leak were not observed except for the patient with ovarian cancer had low grade fever on 2nd day of insertion and was managed conservatively with intravenous antibiotic (Table 2). Late complications like blockage, fractures, dislodgement were not noted. Port pocket infection with collection was seen in one patient for which port device had to be removed on $8^{\text {th }}$ day of implantation. 


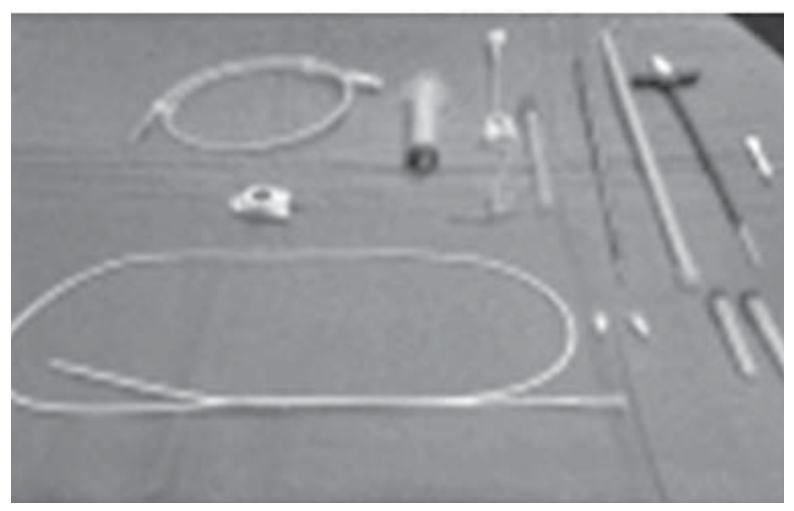

Figure 1: Components of Subcutaneous Venous Access Device

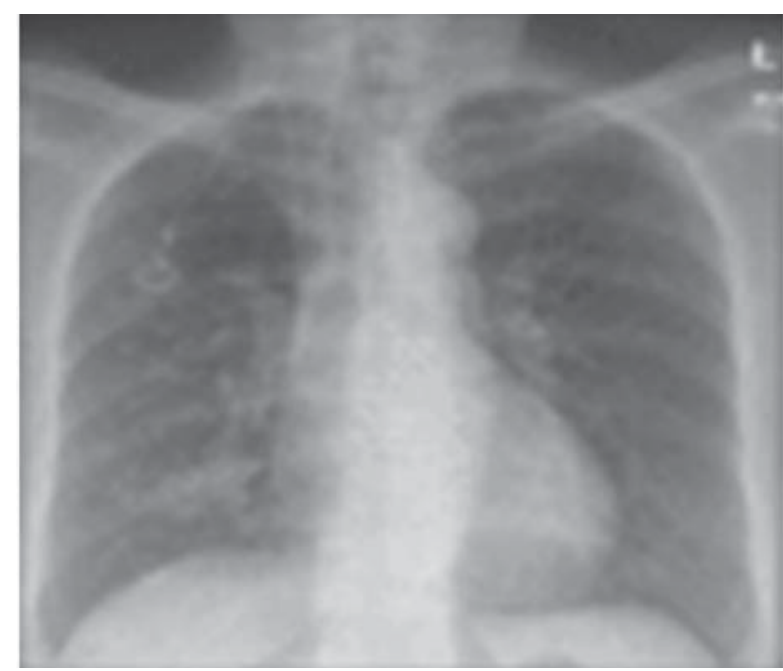

Figure 3: Postprocedural chest X-ray

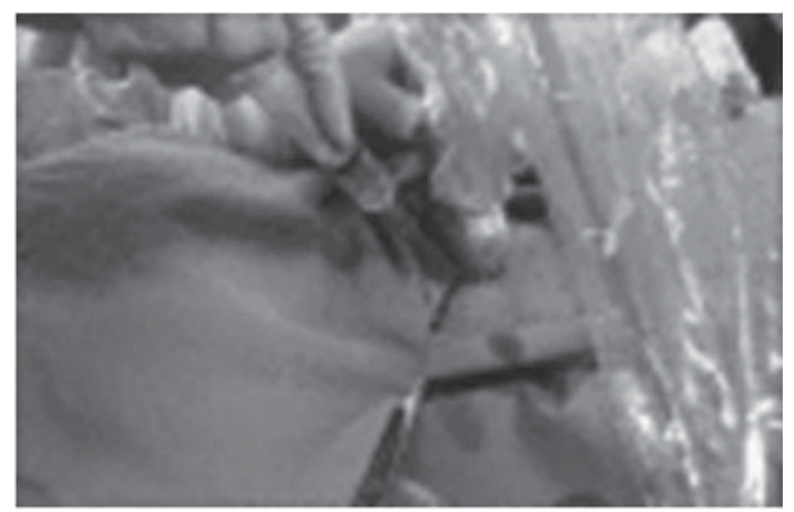

Figure 2: The ultrasound guided in internal jugular vein puncture

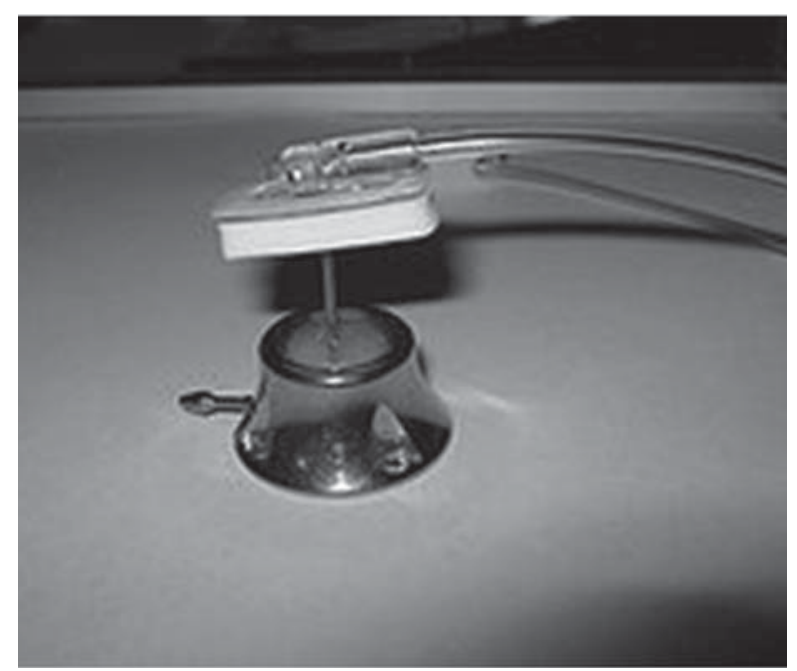

Figure 4: Titanium chemoport

\section{Table 1: Characteristics of total 10 patients on SVAD and total cost of SVAD}

\begin{tabular}{|c|c|c|c|c|c|c|c|}
\hline Age (years) & Sex & Disease & $\begin{array}{l}\text { Port access } \\
\text { (route) }\end{array}$ & Radiological aid & $\begin{array}{l}\text { Pre- } \\
\text { operative } \\
\text { prophylaxis }\end{array}$ & $\begin{array}{l}\text { Operative } \\
\text { time } \\
\text { (minutes) }\end{array}$ & $\begin{array}{l}\text { Cost } \\
\text { (Nepalese } \\
\text { rupees) }\end{array}$ \\
\hline \multirow[t]{2}{*}{ Mean : 50.5} & Male: 6 & Carcinoma & Subcutaneous & Ultrasonography & $1 \mathrm{gm}$ & Mean: 41 & Port device: \\
\hline & Female: 4 & Ovary:4 & Right Internal & Fluoroscopy & Ceftriaxone & & 45000 \\
\hline \multirow[t]{6}{*}{ Range: $25-62$} & & Colon:3 & Jugular Vein & & & & \\
\hline & & Testis:1 & & & & & Operative \\
\hline & & Urinary & & & & & charge: 5000 \\
\hline & & Bladder:1 & & & & & \\
\hline & & Non-Carcinoma & & & & & \\
\hline & & Porphyria:1 & & & & & \\
\hline
\end{tabular}


Table 2: Complication after SVAD implantation

\begin{tabular}{lll}
\hline Per-operative & Early & Late \\
\hline Bleeding : None & Infection : None & Blockage : None \\
\hline Failure :None & Blockage :None & Fracture : None \\
\hline Pneumothorax : None & Thrombosis : None & Dislodgement : None \\
\hline Breakage : None & Leak: None & Port-pocket infection: 1 \\
\hline Displacement : None & Bleeding : None & \\
\hline Arrhythmia : None & Fever :1 \\
\hline Mortality (till 30 days of implantation) : None & & \\
Complication rate & & \\
\hline Early: $1 / 10(10 \%)$ & & \\
Late: $1 / 10(10 \%)$ & \\
Thrombotic complication: $0 / 10(0 \%)$ & \\
Non-thrombotic malfunction: $0 / 10(0 \%)$ &
\end{tabular}

\section{DISCUSSION}

The preliminary case on insertion of SVAD is our pioneer experience to be reported from Nepal. Insertion of SVAD requires surgical expertise and training for proper insertion and good outcome. SVAD insertion is widely popular in western countries and with good patient compliance. The cost of these device, need of surgical expertise, patient's willingness has been major factors of not getting these devices though they have many advantages.

Neiderhuber et al first reported SVAD placement in $1982^{7}$. SAVD has gained wide acceptance for the patients undergoing repeated intravenous chemotherapy. SAVD is safe, long-lasting and satisfactory quality of life as well as cosmetic results ${ }^{8,9}$. Apart from its use in Oncology it has also been adopted for the use to withdraw blood in patients who require frequent blood tests, in hemodialysis patients, to deliver antibiotics to patients and moreover to administer analgesics to cancer patients with chronic pain, and those with sickle-cell disease. The chemoport insertion is performed under the guidance of (USG) and fluoroscopy using the Seldinger technique ${ }^{3}$.

Evaluation of the patency and the size of vessels can be done by ultrasonography with clear visualization before the puncture resulting in high procedural success rate of nearly $100 \%{ }^{2,6,8}$.

Likewise, fluoroscopy helps in localisation of the tip of the catheter and the course of a catheter with quick evaluation of port function. IJV was our first choice than the subclavian vein (SCV). SCV is less preferred for its complications and procedure time. Complications like arterial puncture or pneumothorax are encountered with technical difficulties during advancement of catheter into the brachiocephalic vein. Mal-positioning of a catheter has been encountered due to an acute angle of SCV and brachiocephalic vein ${ }^{8,9}$.

The risk of venous thrombosis is low as catheter traverses in straight course without injuring the vessel wall due to straight course of internal jugular vein to the superior vena cava. Complications such as pneumothorax or pinch-off syndrome due to nerve plexus injury occur only in SCV access ${ }^{8,9}$ is less as compared to SVC insertion as the internal jugular vein (IJV) is located away from the lung or nerve plexus.

Se Jin Ahn et al reported the complication of $4.85 \%$ in 1254 port implantation and similarly the largest series of 8156 port placements by Moureau et al reported total complication rate of 0.52 per 1000 catheter days ${ }^{2,11}$.

Another recent large study by Teichgräber et al reported total complication rate of $11.8 \%$ in 3160 SVAD implantations ${ }^{3}$.

Se JinAhn et al observed 56 post-procedural complications in their study. Complications like infection $(0.6 \%)$, thrombotic malfunction $(1.4 \%)$, nonthrombotic malfunction $(0.9 \%)$, venous thrombosis $(0.5 \%)$, as well as wound problems $(1.1 \%)$ were observed. Thirty six SVAD (3\%) were removed due to complications ${ }^{2}$. The most common complications as reported by Se Jin Ahn et al were blood stream infection $(n=134 ; 5.1 \%)$, catheterinduced venous thrombosis $(n=97 ; 3.7 \%)$ and catheter migration $(n=34 ; 1.3 \%)^{2}$. Likewise, Gebauer et $\mathrm{al}^{10}$ reported a total of 374 (11.8\%) adverse events. Of these adverse events, forty two (1.33\%) were periprocedural, 
eighty six (3.3\%) were early and 246 (9.4\%) were late onset complications after port implantation.

Our entire patients underwent ultrasonography guided puncture of internal jugular vein and placement of port catheter confirmed on fluoroscopy. SVAD was implanted for patients needing intravenous chemotherapy and non-cancer patient requiring repeated intravenous medication. No insertion related complications were observed in our cases. Mild early complication like fever was observed in one patient who was managed conservatively without the need of admission or removal of port. Port removal was needed in another patient due to port pocket infection and collection after $8^{\text {th }}$ day of implantation. The patient had bacteraemia with no untoward life threatening septicaemia. The patient fared well on intravenous antibiotic.

The limitation of our study is for its less number of cases for which complication rate as such cannot be commented however, thrombotic complication (0\%) and non-thrombotic malfunction $(0 \%)$ were not seen in all cases (Table 2). We observed mild perioperative side-effects which were managed conservatively without serious life-threatening conditions.Another

\section{REFERENCES}

1. Yaacob Y, Nguyen DV, Mohamed Z, Ralib AR, Zakaria $\mathrm{R}$, MudaS.Image-guided chemoport insertion by interventional radiologists: A single-center experience on periproceduralcomplications.Indian J Radiol Imaging. 2013 Apr;23(2):121-5.

2. Se JinAhn, MD, Hyo-Cheol Kim, MD, JinWook Chung, MD, Sang Bu An, MD, Yong Hu Yin, Hwan Jun Jae, MD, and Jae Hyung Park, MD. Ultrasound and Fluoroscopy-Guided Placement of Central Venous Ports via Internal Jugular Vein: Retrospective Analysis of 1254 Port Implantations at a Single Center.Korean J Radiol. 2012 May-Jun;13(3):314-323.

3. Teichgräber UK, Kausche S, Nagel SN, Gebauer B. Outcome analysis in 3,160 implantations of radiologically guided placements of totally implantable central venous port systems. EurRadiol 2011;21:1224-1232.

4. Teichgräber UK, Kausche S, Nagel SN.Evaluation of radiologically implanted central venous port systems explanted due to complications.JVasc Access. 2011 Oct-Dec;12(4):306-12.

5. Koroglu M, Demir M, Koroglu BK, Sezer MT, Akhan $\mathrm{O}$, Yildiz $\mathrm{H}$, et al. Percutaneous placement of central venous catheters: comparing the anatomical landmark method with the radiologically guided reason is that patients could not be convinced for use of chemoport to the high due cost of the device, though being advantageous for the disease management and long term placement up to two years. For a cancer patient spending another NRs 50,000 was seen as a huge burden to already economically stressed patients who have already spent significant amount for their own treatment in surgery or chemotherapy (Table 1). Many of the referred cases refused to go for SVAD but instead preferred for PIC line (Peripherally Inserted Central line). Though PIC line is not suitable beyond three months but the cost is half of SAVD of about NRs 25000. One mortality seen before 30 days in the porphyric patient succumbed to his medical cause but not due to catheter related complication.

\section{CONCLUSION}

Chemoport insertion is safe with acceptable mild perioperative complications. Ultrasound guided puncture and fluoroscopic confirmation aided in safe placement of port device. Major drawback for chemoport implantation is the high cost of the device for most financially destabilized cancer patients. However, larger number of cases will give better statistical values in terms of complication rate.

technique for central venous catheterization through the internal jugular vein in emergent hemodialysis patients. ActaRadiol 2006;47:43-47.

6. Randolph AG, Cook DJ, Gonzales CA, Pribble CG. Ultrasound guidance for placement of central venous catheters: a meta-analysis of the literature. Crit Care Med 1996;24:2053-2058.

7. Niederhuber JE, Ensminger W, Gyves JW, Liepman M, Doan K, Cozzi E. Totally implanted venous and arterial access system to replace external catheters in cancer treatment. Surgery 1982;92:706-712.)

8. Dede D, Akmangit I, Yildirim ZN, Sanverdi E, Sayin B. Ultrasonography and fluoroscopy-guided insertion of chest ports. Eur J SurgOncol 2008;34:1340-1343.

9. Kurul S, Saip P, Aydin T. Totally implantable venousaccess ports: local problems and extravasation injury. Lancet Oncol 2002;3:684-692

10. Gebauer B, El-Sheik M, Vogt M, Wagner HJ. Combined ultrasound and fluoroscopy guided port catheter implantation--high success and low complication rate. Eur J Radiol 2009;69:517-522.

11. Moureau N, Poole S, Murdock MA, Gray SM, Semba $\mathrm{CP}$. Central venous catheters in home infusion care: outcomes analysis in 50,470 patients. J VasclntervRadiol 2002;13:1009-1016. 